\title{
Removal Methyl Orange from Aqueous Solutions Using Dragon Fruit (Hylocereusundatus) Foliage.
}

\begin{abstract}
In this study, dragon fruit foliage was used as an adsorbent for the removal of methyl orange from an aqueous solution. The influence of the initial dye concentration, contact time, $\mathrm{pH}$, temperature, dosage of biosorbent and ion strength were investigated in batch experiments. The adsorption was evaluated using Freundlich and Langmuir isotherm models and found to fit the Freundlich model. The kinetic study data were well-represented by the pseudo second order kinetic model. Different thermodynamic values were calculated and the adsorption process was found to be favourable and endothermic. The adsorbent was characterised using Fourier transform infrared spectroscopy and scanning electron microscopy. Results suggest the potential of dragon fruit foliage as an alternative low cost and environmental friendly biosorbent for removal of methyl orange.
\end{abstract}

Keyword: Adsorption; Methyl orange; Dragon fruit foliage; Kinetics; Equilibrium; Thermodynamics 